\title{
The applicability of the Law of Extensible Minors to determinants of special form.
}

\section{By Thomas Muir, LL.D.}

1. In a paper* published in 1897 it was suggested that the Law of Extensible Minors was applicable to Kronecker's linear relation between the $n$-line minors of an axisymmetric determinant of the $(2 n)^{\text {th }}$ order, the concluding sentence of the passage being-" For example, knowing from Kronecker that

$$
\left|\begin{array}{l}
123 \\
456
\end{array}\right|-\left|\begin{array}{l}
124 \\
356
\end{array}\right|+\left|\begin{array}{l}
125 \\
346
\end{array}\right|-\left|\begin{array}{l}
126 \\
345
\end{array}\right|=0
$$

if the terms be minors of the axisymmetric determinant

$$
\left|\begin{array}{llllll}
1 & 2 & 3 & 4 & 5 & 6 \\
1 & 2 & 3 & 4 & 5 & 6
\end{array}\right|_{r, s=s, r},
$$

we can at once vouch for the identity

$$
\left|\begin{array}{l}
12378 \ldots \\
45678 \ldots
\end{array}\right|-\left|\begin{array}{c}
12478 \ldots \\
35678 \ldots
\end{array}\right|+\left|\begin{array}{c}
12578 \ldots \\
34678 \ldots
\end{array}\right|-\left|\begin{array}{c}
12678 \ldots \\
34578 \ldots
\end{array}\right|=0
$$

in connection with the axisymmetric determinant

$$
\left|\begin{array}{lllllllll}
1 & 2 & 3 & 4 & 5 & 6 & 7 & 8 & \ldots \\
1 & 2 & 3 & 4 & 5 & 6 & 7 & 8 & \ldots
\end{array}\right|_{r, s=s, r} \text { " }
$$

No proof was given, as the matter turned up quite incidentally, and verification of the cases made use of was all that was necessary.

I propose now to supply the requisite proof, and to show that the law is applicable also to determinants of another special form.

2. The original proof of the law, as used in connection with a theorem which is its own complementary, consists essentially in applying the complementary of the theorem to the adjugate of an "extension" of the original determinant, and then using Jacobi's

* Murr, T.-The automorphic linear transformation of a quadric. Trans. actions Roy. Soc. Edinburgh, XXXIX. [pp. 209-230] p. 226. 
theorem regarding any minor of the adjugate. Thus, the minors of the first two rows of the determinant $\left|a_{1} b_{2} c_{3} d_{4}\right|$ being connected by the relation

$$
\left|\begin{array}{ll}
a_{1} & a_{2} \\
b_{1} & b_{2}
\end{array}\right| \cdot\left|\begin{array}{ll}
a_{3} & a_{4} \\
b_{3} & b_{4}
\end{array}\right|-\left|\begin{array}{ll}
a_{1} & a_{3} \\
b_{1} & b_{3}
\end{array}\right| \cdot\left|\begin{array}{ll}
a_{2} & a_{4} \\
b_{2} & b_{4}
\end{array}\right|+\left|\begin{array}{ll}
a_{1} & a_{4} \\
b_{1} & b_{4}
\end{array}\right| \cdot\left|\begin{array}{ll}
a_{2} & a_{3} \\
b_{2} & b_{3}
\end{array}\right|=0
$$

we take the complementary theorem

$$
\left|\begin{array}{cc}
c_{3} & c_{4} \\
d_{3} & d_{4}
\end{array}\right| \cdot\left|\begin{array}{ll}
c_{1} & c_{2} \\
d_{1} & d_{2}
\end{array}\right|-\left|\begin{array}{cc}
c_{2} & c_{4} \\
d_{2} & d_{4}
\end{array}\right| \cdot\left|\begin{array}{cc}
c_{1} & c_{8} \\
d_{1} & d_{3}
\end{array}\right|+\left|\begin{array}{ll}
c_{2} & c_{3} \\
d_{2} & d_{3}
\end{array}\right| \cdot\left|\begin{array}{ll}
c_{1} & c_{4} \\
d_{1} & d_{4}
\end{array}\right|=0
$$

and apply it to the array

$$
\begin{array}{cccc}
\mathrm{C}_{1}, & \mathrm{C}_{2}, & \mathrm{C}_{3}, & \mathrm{C}_{4} \\
\mathrm{D}_{1}, & \mathrm{D}_{2}, & \mathrm{D}_{3}, & \mathrm{D}_{4}
\end{array}
$$

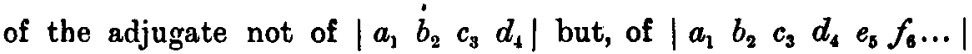
or $\Delta_{n}$ say, thus obtaining

$$
\left|\begin{array}{ll}
\mathrm{C}_{3} & \mathrm{C}_{4} \\
\mathrm{D}_{3} & \mathrm{D}_{4}
\end{array}\right| \cdot\left|\begin{array}{ll}
\mathrm{C}_{1} & \mathrm{C}_{2} \\
\mathrm{D}_{1} & \mathrm{D}_{2}
\end{array}\right|-\left|\begin{array}{ll}
\mathrm{C}_{2} & \mathrm{C}_{4} \\
\mathrm{D}_{2} & \mathrm{D}_{4}
\end{array}\right| \cdot\left|\begin{array}{ll}
\mathrm{C}_{1} & \mathrm{C}_{3} \\
\mathrm{D}_{1} & \mathrm{D}_{3}
\end{array}\right|+\left|\begin{array}{cc}
\mathrm{C}_{2} & \mathrm{C}_{3} \\
\mathrm{D}_{2} & \mathrm{D}_{3}
\end{array}\right| \cdot\left|\begin{array}{cc}
\mathrm{C}_{1} & \mathrm{C}_{4} \\
\mathrm{D}_{1} & \mathrm{D}_{4}
\end{array}\right|=0 .
$$

Then from this, by using Jacobi's theorem, there is derived

and therefore

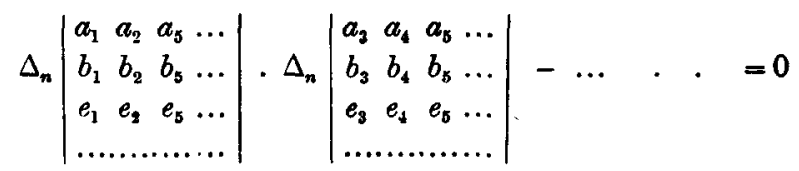

$$
\begin{aligned}
\left|a_{1} b_{2} e_{5} f_{6} \ldots\right| \cdot\left|a_{3} b_{4} e_{3} f_{6} \ldots\right| & -\left|a_{2} b_{3} e_{5} f_{6} \ldots\right| \cdot\left|a_{2} b_{4} e_{5} f_{6} \ldots\right| \\
& +\left|a_{1} b_{4} e_{5} f_{6} \ldots\right| \cdot\left|a_{2} b_{3} e_{5} f_{6} \ldots\right|=0,
\end{aligned}
$$

which is what the original identity becomes if we append to each minor the "extension" $e_{3} f_{6} \ldots$

3. Now if the identity whose Extensional is wanted be true only of a special form of determinant it is clear that this mode of proof will require $(a)$ that the extended determinant be of the same special form as the original and contain the latter as a minor, and (b) that the adjugate be a determinant of the same special form as the original.

These requirements are met in the case of axisymmetric determinants: hence for Kronecker's relation between minors of such a determinant there exists an Extensional. The best mode of expressing the latter will be seen on examining a special case. 
Taking as our original the determinant

we heve from Kronecker

$$
\left|\begin{array}{llll}
a_{1} & a_{2} & a_{3} & a_{4} \\
a_{2} & b_{2} & b_{3} & b_{4} \\
a_{3} & b_{3} & c_{3} & c_{4} \\
a_{4} & b_{4} & c_{4} & d_{4}
\end{array}\right| \text {, or } \Delta_{4} \text { say, }
$$

$$
\begin{aligned}
& \left|\begin{array}{ll}
a_{3} & a_{4} \\
b_{3} & b_{4}
\end{array}\right|-\left|\begin{array}{ll}
a_{2} & a_{4} \\
b_{3} & c_{4}
\end{array}\right|+\left|\begin{array}{ll}
a_{2} & a_{3} \\
b_{4} & c_{4}
\end{array}\right|=0, \\
& \text { or }\left|\begin{array}{ll}
1 & 2 \\
3 & 4
\end{array}\right|-\left|\begin{array}{ll}
1 & 3 \\
2 & 4
\end{array}\right|+\left|\begin{array}{ll}
1 & 4 \\
2 & 3
\end{array}\right|=0 \text {, }
\end{aligned}
$$

the Complementary of which is itself. Applying this theorem to the minor

of the adjugate of

$$
\left|\begin{array}{llll}
\mathbf{A}_{1} & \mathbf{A}_{2} & \mathbf{A}_{3} & \mathbf{A}_{4} \\
\mathbf{A}_{2} & \mathbf{B}_{2} & \mathbf{B}_{3} & \mathbf{B}_{4} \\
\mathbf{A}_{3} & \mathbf{B}_{3} & \mathrm{C}_{3} & \mathbf{C}_{4} \\
\mathbf{A}_{4} & \mathbf{B}_{4} & \mathrm{C}_{4} & \mathrm{D}_{4}
\end{array}\right|
$$

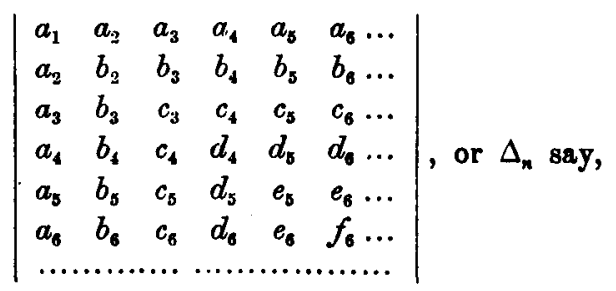

we obtain

$$
\left|\begin{array}{ll}
A_{3} & A_{4} \\
B_{3} & B_{4}
\end{array}\right|-\left|\begin{array}{ll}
A_{2} & A_{4} \\
B_{3} & C_{4}
\end{array}\right|+\left|\begin{array}{ll}
A_{2} & A_{3} \\
B_{4} & C_{4}
\end{array}\right|=0
$$

The use of Jacobi's theorem alters this into

whence in regard to $\Delta_{n}$ we have

$$
\begin{aligned}
\Delta_{n} \cdot \text { compl. of }\left|\begin{array}{ll}
a_{3} & a_{4} \\
b_{3} & b_{4}
\end{array}\right| \text { in } \Delta_{n}-\Delta_{n} \cdot \text { compl. of }\left|\begin{array}{ll}
a_{2} & a_{4} \\
b_{3} & c_{4}
\end{array}\right| \text { in } \Delta_{n} \\
+\Delta_{n} \cdot \text { compl. of }\left|\begin{array}{ll}
a_{2} & a_{3} \\
b_{4} & c_{4}
\end{array}\right| \text { in } \Delta_{n}=0 ;
\end{aligned}
$$

$$
\text { compl. of }\left|\begin{array}{ll}
1 & 2 \\
3 & 4
\end{array}\right| \text { - compl. of }\left|\begin{array}{ll}
1 & 3 \\
2 & 4
\end{array}\right|+\text { compl. of }\left|\begin{array}{ll}
1 & 4 \\
2 & 3
\end{array}\right|=0
$$

or

$$
\begin{array}{ll}
1 & 2 \\
3 & 4
\end{array}:-\begin{array}{ll}
1 & 3 \\
2 & 4
\end{array}:+\begin{array}{ll}
1 & 4 \\
2 & 3
\end{array}:=0 .
$$


Similarly Kronecker's identity

$$
\left|\begin{array}{lll}
1 & 2 & 3 \\
4 & 5 & 6
\end{array}\right|-\left|\begin{array}{lll}
1 & 2 & 4 \\
3 & 5 & 6
\end{array}\right|+\left|\begin{array}{lll}
1 & 2 & 5 \\
3 & 4 & 6
\end{array}\right|-\left|\begin{array}{lll}
1 & 2 & 6 \\
3 & 4 & 5
\end{array}\right|=0
$$

regarding an axisymmetric determinant of the sixth order leads to the identity

$$
\begin{aligned}
& 123 \\
& 456
\end{aligned}-\begin{aligned}
& 124 \\
& 356
\end{aligned}+\begin{aligned}
& 125 \\
& 456
\end{aligned}
$$

which includes Kronecker's and also holds in regard to any axisymmetric determinant of an order higher than the sixth. The latter identity is that given in another form in the sentence quoted in $\$ 1$.

The general result is-If each n-line minor in any Kronecker's relation be written in the form

$$
\left|\begin{array}{lll}
a, & b, & c, \ldots \\
\alpha, & \beta, & \gamma, \ldots
\end{array}\right|
$$

where $a, b, c, \ldots$ are the numbers of the rows and $a, \beta, \gamma, \ldots$ the numbers of the columns which the minor occupies in the original axisymmetric determinant of the $(2 \mathrm{n})^{\text {th }}$ order, then a generalisation (the Extensional) is obtained by changing

$$
\left|\begin{array}{l}
a, b, c, \ldots \\
a, \beta, \gamma, \ldots
\end{array}\right| \text { into } \quad \begin{aligned}
& a, b, c, \ldots \\
& a, \beta, \gamma, \ldots
\end{aligned}
$$

where the numbers now denote omitted lines in an axisymmetric determinant of any order higher than the $(2 \mathrm{n}-1)^{\text {th }}$.

4. In the case of another class of special determinants, viz., centrosymmetric determinants, the requirements specified in the opening sentence of $\$ 3$ are also met, although the fact may not at first be quite evident. The centrosymmetry of the adjugate will readily be admitted: all that requires to be pointed out is that the determinant must here be extended, not by putting the additional lines after the original lines, but by making them occupy a middle position with half the original lines before them and the other half after : thus the four-line centrosymmetric determinant

$$
\left|\begin{array}{llll}
a_{1} & a_{2} & a_{3} & a_{4} \\
b_{1} & b_{2} & b_{3} & b_{4} \\
b_{4} & b_{3} & b_{2} & b_{1} \\
a_{4} & a_{3} & a_{2} & a_{1}
\end{array}\right|
$$


is extended into

$$
\left|\begin{array}{llllll}
a_{1} & a_{2} & a_{5} & a_{6} & a_{3} & a_{4} \\
b_{1} & b_{2} & b_{5} & b_{6} & b_{3} & b_{4} \\
c_{1} & c_{2} & c_{5} & c_{6} & c_{3} & c_{4} \\
c_{4} & c_{3} & c_{6} & c_{3} & c_{2} & c_{1} \\
b_{4} & b_{3} & b_{6} & b_{5} & b_{2} & b_{1} \\
a_{4} & a_{3} & a_{6} & a_{5} & a_{2} & a_{1}
\end{array}\right|
$$

the former being the minor $\begin{array}{lll:}3 & 4 \\ 3 & 4\end{array}:$ of the latter instead of the

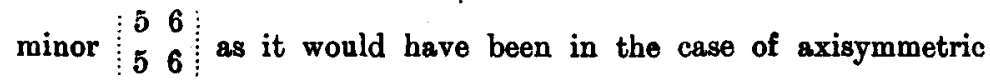
determinants. This being agreed, it follows that there exists an Extensional of the known relation between $n$-line minors of such a determinant of the $(2 n)^{\text {th }}$ order.

Taking the case where $n=4$, viz., the case where the determinant is

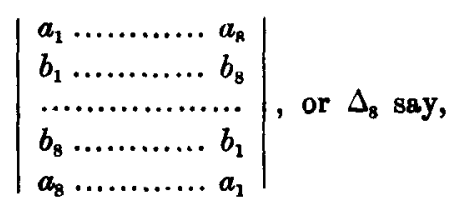

we have as one of the said known identities

$$
\begin{aligned}
& \left|\begin{array}{llll}
1 & 2 & 3 & 4 \\
8 & 2 & 3 & 4
\end{array}\right|+\left|\begin{array}{llll}
1 & 2 & 3 & 4 \\
1 & 7 & 3 & 4
\end{array}\right|+\left|\begin{array}{llll}
1 & 2 & 3 & 4 \\
1 & 2 & 6 & 4
\end{array}\right|+\left|\begin{array}{llll}
1 & 2 & 3 & 4 \\
1 & 2 & 3 & 5
\end{array}\right| \\
= & \left|\begin{array}{llll}
8 & 2 & 3 & 4 \\
1 & 2 & 3 & 4
\end{array}\right|+\left|\begin{array}{llll}
1 & 7 & 3 & 4 \\
1 & 2 & 3 & 4
\end{array}\right|+\left|\begin{array}{llll}
1 & 2 & 6 & 4 \\
1 & 2 & 3 & 4
\end{array}\right|+\left|\begin{array}{llll}
1 & 2 & 3 & 5 \\
1 & 2 & 3 & 4
\end{array}\right| .
\end{aligned}
$$

If we insert $n$ new columns and $n$ new rows into $\Delta_{8}$ in the manner above indicated, calling the new determinant $\Delta_{n+8}$, the very same identity viewed in connection with $\Delta_{n+8}$ must be written

$$
\begin{aligned}
& \left|\begin{array}{cccc}
1 & 2 & 3 & 4 \\
8+n & 2 & 3 & 4
\end{array}\right|+\left|\begin{array}{cccc}
1 & 2 & 3 & 4 \\
1 & 7+n & 3 & 4
\end{array}\right|+\left|\begin{array}{cccc}
1 & 2 & 3 & 4 \\
1 & 2 & 6+n & 4
\end{array}\right|+\left|\begin{array}{cccc}
1 & 2 & 3 & 4 \\
1 & 2 & 3 & 5+n
\end{array}\right| \\
& =\left|\begin{array}{cccc}
8+n & 2 & 3 & 4 \\
1 & 2 & 3 & 4
\end{array}\right|+\left|\begin{array}{cccc}
1 & 7+n & 3 & 4 \\
1 & 2 & 3 & 4
\end{array}\right|+\left|\begin{array}{cccc}
1 & 2 & 6+n & 4 \\
1 & 2 & 3 & 4
\end{array}\right|+\left|\begin{array}{cccc}
1 & 2 & 3 & 5+n \\
1 & 2 & 3 & 4
\end{array}\right| \text {, }
\end{aligned}
$$

as the numbers of the rows and columns beyond the $4^{\text {th }}$ have by the "extension" been increased by $n$. Applying this to the adjugate of $\Delta_{n+8}$, then using Jacobi's theorem regarding a minor of the 
adjugate, and finally removing the power of $\Delta_{n+8}$ which appears as a common factor, we have

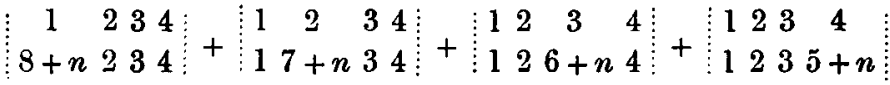

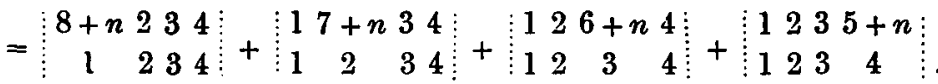

The general result is-If each term of any one of any vanishing aggregates of $\mathrm{n}$-line minors belonging to a centro-symmetric determinant of the $(2 \mathrm{n})^{\mathrm{in}}$ order be written in the form

$$
\left|\begin{array}{lrr}
a, & b, & c, \ldots . . \\
\alpha, & \beta, & \gamma, \ldots \ldots
\end{array}\right|
$$

where $a, b, c, \ldots$ are the numbers of the rows and $a, \beta, \gamma, \ldots$ the numbers of the columns which the minor occupies in the parent determinant, then a generalisation (the Extensional) is obtained by changing

$$
\left|\begin{array}{l}
a, b, c, \ldots \\
a, \beta, \gamma, \ldots
\end{array}\right| \text { into } \quad \vdots a, b, c, \ldots
$$

and increasing by $\mathrm{n}$ each number in the latter that is already greater than $\mathrm{n}$, it being understood that the numbers now denote-omitted lines in a centrosymmetric determinant of any order higher than the $(2 \mathrm{n}-1)^{t h}$. 